\title{
Research on Self-Organization in Resilient Recovery of Cluster Supply Chains
}

\author{
Liang Geng, ${ }^{1,2}$ Renbin Xiao, ${ }^{1}$ and Shanshan Xie ${ }^{1}$ \\ ${ }^{1}$ Institute of Systems Engineering, Huazhong University of Science and Technology, Wuhan 430074, China \\ ${ }^{2}$ School of Science, Hubei University of Technology, Wuhan 430068, China \\ Correspondence should be addressed to Renbin Xiao; rbxiao@hust.edu.cn
}

Received 27 June 2013; Revised 11 September 2013; Accepted 25 September 2013

Academic Editor: Tinggui Chen

Copyright (c) 2013 Liang Geng et al. This is an open access article distributed under the Creative Commons Attribution License, which permits unrestricted use, distribution, and reproduction in any medium, provided the original work is properly cited.

\begin{abstract}
An effective way to deal with high-risk and low-probability disruptions is to create a resilient cluster supply chain, in which the study of resilience lies in its recovery mechanism when failures occur. First, the paper describes the representation method of cluster supply chain resilience. Second, a cluster supply chain network structure generation model is proposed. And based on cascading effect model, it makes analysis of dynamic evolution process when cluster supply chain failure happens. Then it focuses on the self-organization characteristic, which contributes to cluster supply chain emergence overall resilient recovery through local self-organization reconstruction behavior. We also make theoretical analysis of cluster supply chain network characteristics and its effect on the resilience, which helps to illustrate that the root of vulnerability lies in cascading failure while self-organization is the key to resilient recovery. Besides, with the study of self-organization characteristic, it provides theoretical guidance for local control and further achievement of overall resilient optimization.
\end{abstract}

\section{Introduction}

With economic globalization, customer needs become more diverse and personalized, and economic operation is facing more challenges and uncertainties, all of which make it a difficult problem for the enterprises to operate. As a new supply chain network organization, cluster supply chain can effectively solve this problem since it combines characteristics of both industry cluster and supply chain. Besides, the network structure of cluster supply chain contributes to its generation of positive coordination effect and innovation effect. However, with the scale increasing, the cluster supply chain needs to suffer more risks to improve efficiency.

Cluster supply chain is faced with internal uncertainty and external unpredictable events. Therefore, cluster supply chain risks can be divided into two categories. (1) The first category is the risk of supply and demand, which is a kind of operational risk caused by internal conflict, such as machine failure, demand uncertainty, human changes, and mainly solved by internal coordination. The frequency of such risks is usually high, while the impact is relatively small. (2)
The second one is the risk caused by external interrupts [1], such as strikes, natural disasters, and even terrorist attacks. It affects the normal flow of the logistics, information, and capital in cluster supply chain or even makes some links cannot normally operate. Under emergencies, a small perturbation or a minor fault in the network can give rise to a pass-through effect on the whole network or even causes cascading failure of the entire network system, resulting in serious consequences. The frequency of such risk is low, while the impact is very serious. In recent years, with such events occurring frequently, supply chain suffers from disruptions or even collapse, which brings about huge economic losses to enterprises. For example, in March 11, 2011, a Richter 8.9 earthquake hit Japan, bringing about more than $\$ 4$ billion direct economic losses to Toyota. Therefore, when facing such interrupts caused by emergencies, the cluster supply chain system is rather vulnerable. In order to overcome the vulnerability, the supply chain system should combine the advantages of both robust strategy and flexible strategy, which means that the system should possess robustness and adaptability at the same time. Furthermore, resilience should 
get more attention. Therefore, the concept of supply chain resilience came into being, based on which the concept of creating resilient supply chain is proposed.

The key to study cluster supply chain resilience is to comprehend recovery mechanism. As the premise of recovery is the occurrence of failures, we should first understand how the failure occurs. Under the disruptions environment, the occurrence of cascading effect is mainly due to the failure of some critical nodes, which brings about serious losses to the cluster supply chain structure or even cripples the whole network operation. At this point, as a complex adaptive system, the resilient cluster supply chain can make quick adjustments and respond to the existing resources according to the current logical constraint conditions of the remaining nodes, which is in accordance with the given principle of self-organization repair, and both new generated nodes and remaining nodes reconstruct logical relationship between each other. Owing to the local self-organization repair behavior of old and new nodes, the supply chain can make a new network structure emerge and restore its overall function quickly. This self-organization repair ability adequately reveals the resilient response strategy of cluster supply chain.

The rest of this paper is organized as follows. Section 2 presents the descriptive approach of cluster supply chain resilience based on the literature review. Section 3 defines a network structure model based on the characteristics of cluster supply chain network. In Section 4, we analyze the causes of cascading failure and the impact of network structure on resilience. In Section 5, we find out that the resilient recovery mechanism of cluster supply chain network lies in its selforganization. It is proved that the key to resilient recovery of cluster supply chain is self-organization with reference to a simulation case in Section 6. Finally, conclusions and possible future research extensions comprise Section 7.

\section{Literature Review}

The concept of "resilience" is first derived from the papers of Holling [2], who defines the resilience as the ability of a system to absorb disturbance before its equilibrium changes. Anderson [3] pointed out that resilience is not just about recovery; it means that the system is flexible enough to adapt to positive or negative impacts. Later, scholars of different disciplines gradually adopt the concept of resilience to describe the key features of complex dynamic systems that they have researched, and different scholars have different definition of the supply chain resilience. Christoper and Pack [4] deemed that the resilience refers to the ability of a system to recover to the original (or better) state after an interruption. Fiksel [5] thought that the resilience is the ability of a system to survive, adapt, and develop when facing disturbance. Rice and Caniato [6] considered that resilience is the ability to deal with undesirable disruptions and restore normal operations. Sheffi [7] thought that resilience is the ability to restrain disruption and recover from it. Based on the above scholars' points, the supply chain resilience just as the word resilience emphasizes is the ability to quickly recover, and also includes both the word elasticity which means adaptability and the word flexibility which means flexibility and antidisruption.

Some scholars have already carried out some qualitative researches on the key theory of the supply chain resilience. For example, Sheffi [8] is an early scholar who has made significant achievements in the aspect of supply chain resilience. He thought the most direct and effective way is to improve supply chain structure. Recently some scholars make quantitative study on supply chain resilience and analyze internal mechanism in-depth, which provides managers with more direct help. Zhao et al. [9], for example analyzed resilience of supply network topology structure under random attacks and attempted attacks from the aspects of availability, connectivity, accessibility, and so forth, which focused on military security network. Huang et al. [10] tried to measure the vulnerability of supply chain nodes with degree centrality, betweenness centrality, and network factions through the use of social network analysis method. Yan et al. [11] studied emergency management strategy of resilient supply chain based on node failure. The research mentioned above studies space resilience of supply chain from topology structure, and the importance of the nodes is equal to node destruction based on the thought of deleting node. Without considering node repair ability, it is unable to reflect time resilience of supply chain, not to mention the recovery ability that resilience owns.

Based on the synthesis of existing literature [12], resilience can be expressed from various aspects as follows.

2.1. Space Resilience. The space resilience of supply chain is defined as a measure of the overall supply chain effect ability in the spatial structure. When resilient supply chain faces an internal or external disruption, there may be great change in its structure, such as node failure, edge failure. In addition, the resilient supply chain should have a self-restoring property, thus active nodes in the network constantly vary with failure and recovery. We adopt the total load change of the active nodes in the network to measure the space resilience of supply chain.

2.2. Time Resilience. The time resilience of resilient supply chain is the measure of the resilient supply chain ability to response and restore in the time dimension. When the resilient supply chain is faced with disruptions, it should be able to recover its response to user and performance level of the supply chain within a certain time range and reach a steady state in a short time. We adopt the total load of the active nodes in the network to express the network performance and use the changes of the performance over time to measure the time resilience of supply chain.

2.3. Resilience Description. The description method of supply chain resilience that presented in this paper is shown in Figure 1, in which time period $0-T_{s}$ reflects response delay time, time period $T_{s}-T_{r}$ reflects destruction spread time, and time period $T_{r}-T_{e}$ reflects recovery time. With next round of failure and recovery going on until the system reaches 


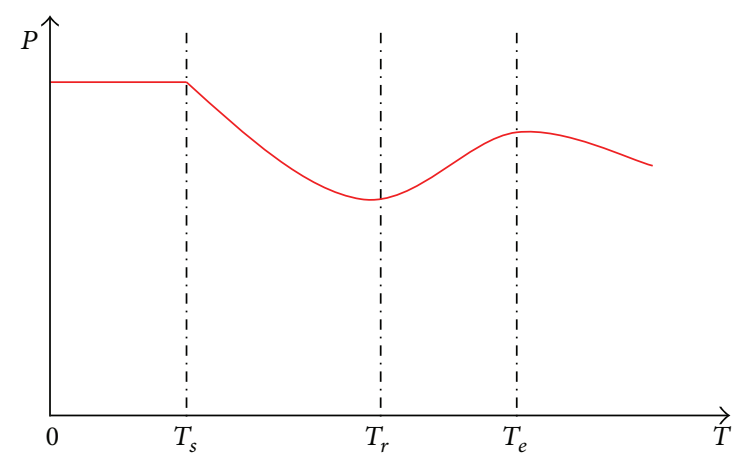

FIGURE 1: Resilience description of cluster supply chain network.

a steady state, the height of the curve reflects the cluster supply chain network performance at time $t$. This kind of cluster supply chain network resilience includes characters of three aspects, which are absorption capacity, adaptability, and recovery ability.

Absorption capacity exhibits anti-interference, which is able to automatically absorb the impact of disturbance at a small price, making the impact of damage achieve minimization and not affect system overall performance, shown as time $0-T_{s}$. Although cascading effect has already began during this period, because of the complementarities of cluster supply chain network, the decline in overall performance does not immediately appear.

Adaptability shows active response, which is the selforganization capacity of each member in the supply chain network and also the self-adjusting ability during damage period and recovery period. Adaptability is shown as curvature degree of the curve during the time period $T_{s}-T_{e}$. The actual supply chain system consists of nodes with selflearning ability [13], which can optimally distribute its load to its adjacent nodes when it fails and also can optimally reobtain load after recovery.

Recovery ability is a kind of ability that is easy to recover to a new stable state, shown as the time period $T_{r}-T_{e}$. The system begins to recover at $T_{r}$, and the change of the network performance within the time period $T_{r}-T_{e}$ reflects recovery ability. Recovery ability of cluster supply chain network members consists of two parts, which are their own recovery ability and recovery ability related to other members.

Such description of cluster supply chain network resilience not only clearly outlines the space characteristics and time characteristics of the cluster supply chain network but also meets the several major features that supply network resilience should have, such as self-recovery, antidisruption, and active response. The resilience framework makes quantitative assessment of supply chain's absorption capacity, adaptability, and recovery ability.

2.4. Definition of Resilience. Cluster supply chain resilience is defined as follows: cluster supply chain network suffers from cascading failure when dealing with undesirable disruption, but it can conduct self-repair through adaptability and make it fast recover to a new stable state.

\section{Cluster Supply Chain Network}

3.1. Concept of Cluster Supply Chain. Cluster supply chain is a kind of supply chain coupling with industrial cluster, and its effective operation plays an important role in the promotion of industrial clusters. Because of the physical proximity and industry relevancy, cluster enterprises have the characteristics of flexibility, specialization, trust, and cooperation. They cooperate and compete with each other in the single-chain as well as among different cross-chains, which makes them adaptive to the rapid change of market demand and enhance international competitiveness. Based on this, supply chains that cause cross-industry competition and cooperation are called "cluster supply chain," the core of which is to enhance the competitiveness of industry cluster.

Cluster supply chain promotes internal labor division and external collaboration. Enterprises provide a variety of products and services and strengthen regional economy through collaboration. Cluster supply chain possesses resource aggregation effect, which can gather some economic resources, such as technology, capital and labor, and promote resource aggregation effectively as well as industrial structure adjustment.

Cluster supply chain has the following advantages [1416]: (1) strengthen mutual trust based on the common interests; (2) strengthen conscious cooperation; (3) closely complement each other; (4) be closely organized, of low cost, lower information communication, and coordination cost; (5) have stronger ability of learning and innovation. For example, the accumulation of specialized suppliers and skilled labor can reduce the factors that affect production costs. With relatively lower cost and risk, cluster supply chain enterprises enhance their pursuit of technology innovation in order to obtain high profits and achievements. Because of mutual trust, any technology innovation success of the member in cluster supply chain will result in the whole cluster supply chain faster response to market changes than outside enterprises. Cluster can generate high levels of innovation.

3.2. Characters of Cluster Supply Chain. The current supply chain research evolved from research about "single supply chain" to research about "cross-network based on multiple supply chain," focusing on the "supply chain network" that centers on core business and its multidistributors as well as its multicustomers. Cluster supply chain is a network of organizations involved in the different processes producing value in the form of products and services for the ultimate consumer. There are many suppliers, manufacturers, and distributors around the same industry or related industry value chain. Cluster supply chain networks are sequentially arranged based on the vertical ties between enterprises in different layers. Network analysis explicitly differentiates between horizontal ties (transactions in the same layer) and vertical ties (transactions between layers) [17], as shown in Figure 2, mapping how enterprises in one layer are related to each other and to enterprises in other layers.

One of the most important features of cluster supply chain is the interaction among enterprises which may take place between similar firms (horizontal) and, to a greater 


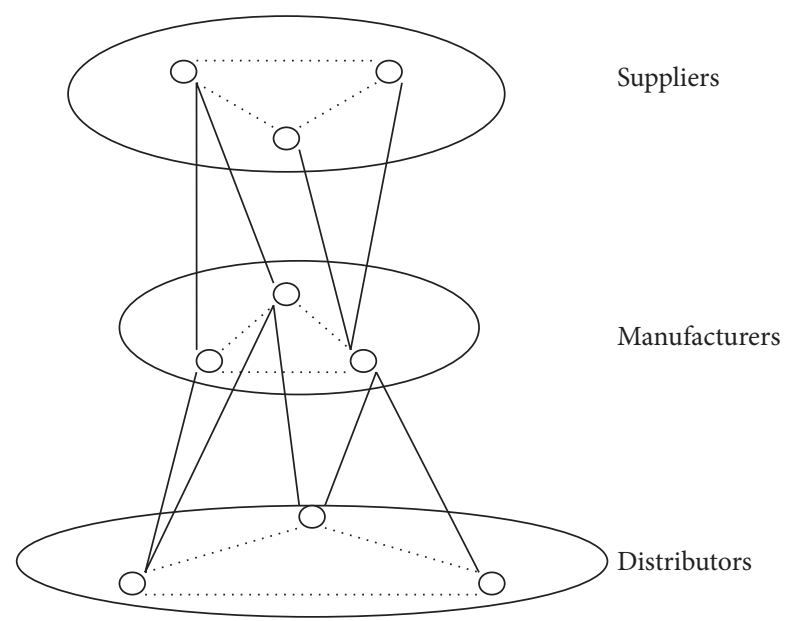

FIGURE 2: Network structure of cluster supply chain.

extent, between enterprises involved in different phases of the production process (vertical). These interactions are manifested in interaction of logistics, capital flow, and information flow between manufacturers, suppliers, and distributors. This interaction makes enterprises in the cluster supply chain technological spillovers: the increase of workers' production skill, operational management and equipment improvement of enterprises, and continuous improvement of market information transfer rate and efficiency. Each enterprise in the cluster can collaborate from the two-dimension (the vertical and the horizontal) to maximize its efficiency.

Cluster supply chain network possesses the characteristic of dynamic evolution. In the fierce competition, it is possible for each node enterprise to be eliminated or rejoin the cluster supply chain network.

Cluster supply chain network is provided with the characteristic of preferential attachment. New connection between nodes in the network is continuously formed and the old connection constantly disappears. The new entrant will select partners based on its demand for resources according to the merits of alternative nodes. Each node enterprise status is arranged in a hierarchy, and it is usually easier for the core enterprise to obtain relatively abundant resources in information, knowledge, reputation, and so forth than those enterprises on the edge of the network. In addition, the core enterprise has an advantage when attracting new entrants because of its relatively higher degree.

The characteristics of cluster supply chain network mentioned above bring an opportunity of resource integration effect for the node enterprise, which also bring risks for the cluster supply chain because of the complexity of the network structure and the complexity of the relationship between cooperative enterprises. The inherent root cause of cluster supply chain network risks lies in the complexity of cluster supply chain topology [18].

This paper presents a new selection mechanism to simulate real situations about cluster supply chain network.

3.3. Structure Model of Cluster Supply Chain Network. Cluster supply chain contains a number of nodes and edges, which form a network structure together. We can use an undirected graph $G=(V, E)$ to represent the network, where $V=\left\{v_{i}\right\}$ is a nonempty finite set of node enterprises in the network, which includes a number of supplier nodes $S_{i}(i=1,2, \ldots, I)$, a number of manufacturer nodes $M_{j}(j=1,2, \ldots, J)$, and a number of distributor nodes $D_{k}(k=1,2, \ldots, K), E=$ $\left\{e_{i j}\right\}$ is a finite set of edges in the network, which represents the various logistics; information flow, capital flow between each enterprise, and the dynamic connections established between each node enterprise are based on the various flows. Adjacency matrix $A_{N \times N}=\left[a_{i j}\right]_{N \times N}$ expresses the business relationship between each logistic node, in which if there is business relationship between any node $v_{i}$ and $v_{j}$, set $a_{i j}=1$, otherwise set $a_{i j}=0$. Degree $k_{i}$ represents the number of other nodes that node $i$ connects, which means the enterprise establishes a relation with other $k_{i}$ enterprises.

Whenever adding a new node enterprise, there will be $m$ old node enterprises to establish business relationship with it. The first step when a node chooses partners is to consider the business connectivity, which means to choose its "local world." And the second step is to make selection in the "local world" formed by business relationships that can be established and establish business relationship.

When a new node enterprise adds into the cluster supply chain network, we should first determine its local world, which means the node enterprise scope that it can establish business relationship with, based on the node enterprise property. If the new node enterprise that joins the network is a supplier, it can establish business relationship with suppliers and manufacturers. If the new node enterprise that joins the network is a manufacturer, it can establish business relationship with suppliers, manufacturers, and distributors. And if the new node enterprise that joins the network is a distributor, it can only establish business relationship with manufacturers.

When choosing partners, mostly prefer to select the enterprise with stronger capacity and the node degree can reflect the node capacity. Suppliers with larger degree reflect that they have a relatively strong supply capacity, manufacturers with larger degree reflect that they have more supply channels and target customers, and distributors with larger degree reflect that they have more purchasing channels and relatively strong purchasing power. So the way of connection is based on the existing nodes and the probability which is proportional to its degree to connect the new node. In other words, the new enterprise tends to establish relationship with large-scale enterprises (nodes with larger degree values).

The new added node selects the appropriate node within its local world according to its preference, and the specific modeling process is as follows:

(1) at the initial time $t=0$, given a randomly generated network with $m_{0}$ nodes and random connections;

(2) add a new node $j$ at each time interval according to a uniform distribution, randomly determine the category of this node, and then select an already existing $m$ nodes to establish connection $\left(m<m_{0}\right)$, where the $m$ nodes must be within the local world $A_{j}$ 
of new node $j$. Use the preferential rules to calculate the probability of connection:

$$
\prod_{\text {local }}=\frac{k_{i}(t)}{\sum_{l \in A_{j}} k_{l}(t)}
$$

(3) select $m$ nodes from $A_{j}$ to connect the new node $j$ according to the connection probability that is calculated in step (2);

(4) loop steps (2), (3) to the preset network size and finally obtain a network with $N$ nodes.

In the evolutionary model, the evolution of the degree $k_{i}$ of node enterprise $i$ over time can be calculated by meanfield approximation method [19]. $k_{i}$ satisfies the following dynamic equation:

$$
\frac{\partial k_{i}}{\partial t}=m \prod^{\prime}\left(i \in A_{j}\right) \prod_{\text {local }}=m \frac{M_{t}}{m_{0}+t} \frac{k_{i}(t)}{\sum_{l \in A_{j}} k_{l}(t)},
$$

where $m$ is the number of edges brought by new node, $M_{t}$ is the number of nodes in the local world of the new node that joins the network at time $t, m_{0}+t$ is the sum of nodes in the network at time $t, \prod^{\prime}\left(i \in A_{j}\right)$ is the probability of node $i$ to be selected into the local world, and $\prod_{\text {local }}$ is the probability for the new node to connect node $i$ in the local world.

To simplify the following analysis, we assume that $\sum_{l \in A_{j}} k_{l}(t)=\left\langle k_{i}\right\rangle M_{t}$, where the average degree is $\left\langle k_{i}\right\rangle=$ $2\left(m t+e_{0}\right) /\left(m_{0}+t\right)$

$$
\begin{gathered}
\sum_{l \in A_{j}} k_{l}(t)=2\left(m t+e_{0}\right) \frac{M_{t}}{m_{0}+t} \approx 2 m M_{t}, \\
\frac{\partial k_{i}}{\partial t} \approx \frac{m M_{t}}{m_{0}+t} \frac{k_{i}(t)}{2 m M_{t}} \approx \frac{k_{i}}{2 t} .
\end{gathered}
$$

Then we can get

$$
\begin{gathered}
k_{i}(t)=m\left(\frac{t}{t_{i}}\right)^{1 / 2}, \\
P(k) \sim 2 m^{2} k^{-3} .
\end{gathered}
$$

This shows that the network model established possesses the characteristic of scale-free and can accurately reflect the realistic cluster supply chain network, in which most of the nodes have just a few adjacent nodes associated with businesses while the minority core enterprise nodes bear large business capacity and need to maintain business contact with a large number of nodes.

\section{Cascading Failure of Cluster Supply Chain}

In the face of unexpected disruptions, the greatest risk of the cluster supply chain is the trigger of cascading effect, which may lead to the collapse of the whole line. The existing research lacks in-depth study of such microscopic mechanism, which is the key to ensure the normal operation of cluster supply chain. Although some scholars have studied cascading failure of the network, most of the works in their research refer to a generic complex network [20] or a specific network, such as the power grid [21], urban infrastructure networks [22], and lacking specific study of the cluster supply chain network. Based on the network model of cluster supply chain, this section proposed a definition method of the initial load based on the degree distribution, using the node load capacity as the load redistribution standards for failing nodes, and further researched the effect of the cluster supply chain network structure on the robustness to resist cascading failure.

4.1. Cascading Failure Mode of Cluster Supply Chain. We proposed a model to represent cascading failures of cluster supply chain. We show that the breakdown of a single node, which causes load redistribution to other nodes, is sufficient to result in the whole system failure.

As we can know from the characteristics of the cluster supply chain network, the load of the cluster supply chain network refers to the operational capacity of the nodes in the network, and the maximum volume of business that each node can carry is the node's load capacity. Load transfer refers to the fact that the business of some nodes transfers to other nodes, including both the transfer of resources and the transfer of the ability.

Different nodes in cluster supply chain network have different loads and different load capacities, and they have different degrees of influence on the overall network when they fail. Therefore, the primary key issue is how to measure the node's load. Node degree is not only able to reflect its network importance but also can reflect the operational capacity of the node, which is the load capacity.

In most previous cascading failure models, the load on a node was generally determined by its degree [23], which may result in the loss of some information. Consider that the adjacent nodes of a node, which have business relationship with it, also affect its load except its own impact. Assume that the initial load $L_{i}(0)$ of node $i$ being dependent on the degree of node $i$ and the degrees of its neighbor nodes, the expression of which is defined as follows:

$$
\begin{gathered}
L_{i}(0)=k_{i}^{\alpha}(0)\left(\sum_{v_{j} \in \Gamma_{i}} k_{j}(0)\right)^{1-\alpha} \\
\left(0 \leq \alpha \leq 1, \quad v_{i}, v_{j} \in V, i \neq j\right),
\end{gathered}
$$

where $k_{i}(0)$ is the degree of node $v_{i}$ at initial time, $\Gamma_{i}$ is the set of adjacent nodes of node $v_{i}$. Parameter $\alpha$ is used to adjust the influence weight of node $v_{i}$ on its own initial load, and $1-\alpha$ is used to accordingly adjust the influence degree of adjacent nodes on the initial load of node $v_{i}$.

We know that each node of cluster supply chain has a limited capacity, which is the largest load that the node can handle. We assume that the load capacity $C_{i}$ of node $i$ is proportional to its initial load:

$$
C_{i}=\beta L_{i 0} \quad(\beta \geq 1),
$$


where $\beta$ is a parameter to adjust the network's overall load bearing capacity. As the parameter value increases, the given load capacity of each node becomes stronger, and the ability to resist the shock that is brought by additional load distribution of the failing node grows stronger. However, because the entity network in actual environment is subject to cost constraints, the greater the load capacity nodes have, the larger the total cost of network investment is. The parameter value of $\beta$, at which the whole system achieves best load bearing capacity and least investment cost, is called critical threshold and assumed as $\beta_{\theta}$. When $\beta=\beta_{\theta}$, the whole network achieves optimal investment cost and optimal network survivability.

As the business capacity of the adjacent nodes has a direct impact on the load redistribution after the node fails, this paper constructed the following load redistribution rules for the failure nodes based on the size of the node degree. Assume that node $v_{i}$ fails, its load will be allocated to its adjacent node $v_{j}$ according to the following proportional function and the proportion is $P_{j}(t)$ :

$$
P_{j}(t)=\frac{k_{j}^{\alpha}(t)\left(\sum_{v_{\mu} \in \Gamma_{j}} k_{\mu}(t)\right)^{1-\alpha}}{\sum_{v_{\eta} \in \Gamma_{i}}\left[k_{\eta}^{\alpha}(t)\left(\sum_{v_{\varphi} \in \Gamma_{\eta}} k_{\varphi}(t)\right)^{1-\alpha}\right]} .
$$

According to the above load redistribution rule, any adjacent node $v_{j}$ will receive a proportion of the failure load distribution $\Delta L_{i \rightarrow j}(t)$, which is

$$
\Delta L_{i \rightarrow j}(t)=\delta L_{i}(t) P_{j}(t) \quad(0 \leq \delta \leq 1) .
$$

The real-time load of the adjacent node $v_{j}$ is

$$
L_{j}(t+1)=L_{j}(t)+\sum_{v_{i} \in \Gamma_{j}} \Delta L_{i \rightarrow j}(t) .
$$

Due to the constraints of node load capacity in cluster supply chain, when the real-time load of a node exceeds its load capacity, this node will result in its own collapse failure, which will lead to a new round of node failure and load redistribution. To effectively describe the dynamic propagation process, the failure propagation function is defined as follows:

$$
L_{j}(t+1)>C_{j} .
$$

In order to show the cascading phenomenon of cluster supply chain, here we focus on cascades triggered by the removal of a node. Assume that any logistics node $v_{a}$ collapses and fails, while its adjacent nodes are $v_{b x}(x=1,2,3)$. Then part of the business load of $v_{a}$ is redistributed to these adjacent nodes (as shown in dashed red arrows). At the same time, remove the edges between node $v_{a}$ and its adjacent nodes $v_{b x}$. This is the first round of node failure and load distribution, as shown in Figure 3.

When the adjacent node $v_{b 1}$ meets the failure transfer function, then the node also collapses and fails, resulting in a new round of failure load redistribution (as shown in dashed green arrows). Meanwhile, remove the edges between node

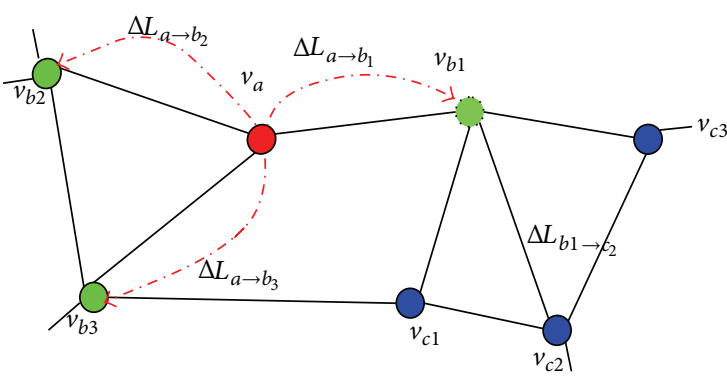

FIGURE 3: The first round of node failure and its load distribution.

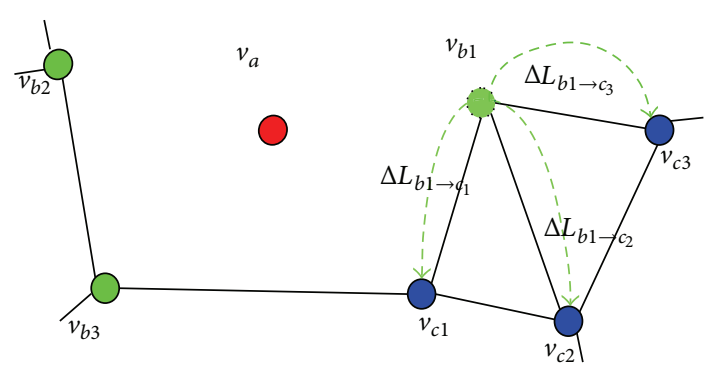

FIGURE 4: The second round of node failure and its load distribution.

$v_{b 1}$ and its adjacent node $v_{c x}$, which is the second round of node failure and load distribution, as shown in Figure 4. As the process carries on, finally the dynamic reaction process of cascading failure will be formed.

4.2. The Impact of the Network Structure on Resilience. Now, we will analyze this model from the perspective of theoretical analysis. First, the given conditional probability $P\left(k^{\prime} \mid k_{i}\right)$ represents the probability that the node of $k^{\prime}$ degree connects the node of $k_{i}$ degree in the network. Then the sum of the degree of any node $v_{i}$ 's adjacent nodes satisfies the following formula:

$$
\sum_{v_{j} \in \Gamma_{i}} k_{j}=\sum_{k^{\prime}=k_{\min }}^{k_{\max }} k_{i} P\left(k^{\prime} \mid k_{i}\right) k^{\prime},
$$

where $k_{\min }$ and $k_{\max }$ are, respectively, the maximum and minimum degree of the nodes in the network. And the nodes degree in the complex network meets the normalization condition:

$$
\sum_{k} P(k)=\sum_{k^{\prime}} P\left(k^{\prime} \mid k\right)=1
$$

Furthermore, consider that the amount of edges from the node of $k$ degree connects to the node of $k^{\prime}$ degree, which will be certainly equal to the amount of edges from the node of $k^{\prime}$ degree that connects to the node of $k$ degree. The degree between nodes should satisfy the following equilibrium conditions:

$$
k P\left(k^{\prime} k\right) P(k)=k^{\prime} P\left(k k^{\prime}\right) P\left(k^{\prime}\right) .
$$


Because the nodes in scale-free network are provided with characteristics of independence on the node, it should meet the following formula:

$$
P\left(k^{\prime} k\right) \cong P\left(k^{\prime}\right) .
$$

Hence, combining formula (13) (15), we can get

$$
\sum_{k} k P\left(k^{\prime} \mid k\right) P(k)=\sum_{k} k^{\prime} P\left(k \mid k^{\prime}\right) P\left(k^{\prime}\right) .
$$

Through simplification, we can get

$$
\begin{aligned}
P\left(k^{\prime}\right) \sum_{k} k P(k) & =k^{\prime} P\left(k^{\prime}\right) \sum_{k} P(k) \\
& \Longrightarrow P\left(k^{\prime}\right)\langle k\rangle \\
& =k^{\prime} P\left(k^{\prime}\right) .
\end{aligned}
$$

Combining formula (14) and formula (17), we can get

$$
P\left(k^{\prime} k\right)=\frac{k^{\prime} P\left(k^{\prime}\right)}{\langle k\rangle} .
$$

As we can know from formula (12) and formula (18)

$$
\begin{aligned}
\sum_{v_{j} \in \Gamma_{i}} k_{j} & =\sum_{k^{\prime}=k_{\min }}^{k_{\max }} k_{i} P\left(k^{\prime} \mid k_{i}\right) k^{\prime} \\
& =k_{i} \sum_{k^{\prime}=k_{\min }}^{k_{\max }} \frac{k^{\prime} P\left(k^{\prime}\right) k^{\prime}}{\langle k\rangle} \\
& =\frac{k_{i}\left\langle k^{2}\right\rangle}{\langle k\rangle} .
\end{aligned}
$$

Therefore, we can derive the following formula:

$$
\begin{aligned}
\sum_{v_{j} \in \Gamma_{i}} k_{j}^{\alpha} & =k_{i} \sum_{k^{\prime}=k_{\min }}^{k_{\max }} \frac{k^{\prime} P\left(k^{\prime}\right) k^{\prime \alpha}}{\langle k\rangle} \\
& =\frac{k_{i}\left\langle k^{1+\alpha}\right\rangle}{\langle k\rangle} .
\end{aligned}
$$

According to the rules of the cascading failure function, if and only if the condition (11) is satisfied, can the network control the occurrence of cascading failure. Combining formula (6), formula (7), and formula (11), therefore

$$
\begin{gathered}
{\left[k_{i}^{\alpha}\left(\sum_{v_{j} \in \Gamma_{i}} k_{j}\right)^{1-\alpha}\right] \cdot \frac{k_{j}^{\alpha}\left(\sum_{v_{\mu} \in \Gamma_{j}} k_{\mu}\right)^{1-\alpha}}{\sum_{v_{\eta} \in \Gamma_{i}}\left[k_{\eta}^{\alpha}\left(\sum_{v_{\varphi} \in \Gamma_{\eta}} k_{\varphi}\right)^{1-\alpha}\right]}} \\
+\left[k_{j}^{\alpha}\left(\sum_{v_{\mu} \in \Gamma_{j}} k_{\mu}\right)^{1-\alpha}\right] \\
<\beta\left[k_{j}^{\alpha}\left(\sum_{v_{\mu} \in \Gamma_{j}} k_{\mu}\right)^{1-\alpha}\right] .
\end{gathered}
$$

Through simplification, we can get

$$
1+\frac{k_{i}^{\alpha}\left(\sum_{v_{j} \in \Gamma_{i}} k_{j}\right)^{1-\alpha}}{\sum_{v_{\eta} \in \Gamma_{i}}\left[k_{\eta}^{\alpha}\left(\sum_{v_{\varphi} \in \Gamma_{\eta}} k_{\varphi}\right)^{1-\alpha}\right]}<\beta .
$$

Therefore, according to the above formula (20), simplify formula (22) to

$$
\begin{aligned}
1+ & \frac{k_{i}^{\alpha}\left(\sum_{v_{j} \in \Gamma_{i}} k_{j}\right)^{1-\alpha}}{\sum_{v_{\eta} \in \Gamma_{i}}\left[k_{\eta}^{\alpha}\left(\sum_{v_{\varphi} \in \Gamma_{\eta}} k_{\varphi}\right)^{1-\alpha}\right]} \\
& =1+\frac{k_{i}^{\alpha}\left(k_{i}\left\langle k^{2}\right\rangle /\langle k\rangle\right)^{1-\alpha}}{\sum_{v_{\eta} \in \Gamma_{i}}\left[k_{\eta}^{\alpha}\left(k_{\eta}\left\langle k^{2}\right\rangle /\langle k\rangle\right)^{1-\alpha}\right]} \\
& =1+\frac{k_{i}}{\sum_{v_{\eta} \in \Gamma_{i}} k_{\eta}}=1+\frac{k_{i}}{k_{i}\left\langle k^{2}\right\rangle /\langle k\rangle} \\
& =1+\frac{\langle k\rangle}{\left\langle k^{2}\right\rangle}<\beta .
\end{aligned}
$$

As we can know from the above mentioned, taking the cost factor into account, the smaller the value of $\beta$, the better. Thus, the optimal load bearing threshold value of $\beta_{\theta}$ of the whole network is shown in the following formula:

$$
\beta_{\theta}=1+\frac{\langle k\rangle}{\left\langle k^{2}\right\rangle} .
$$

Due to the network structure model of cluster supply chain, the degree distribution of the nodes approximately meets the following formula (5), where $m$ is the minimum degree of the nodes in the network; that is, $m=k_{\min }$. Therefore, we can get the following formula:

$$
\begin{aligned}
\left\langle k^{2}\right\rangle & =\int_{k_{\min }}^{k_{\max }} P(k) k^{2} d k \\
& =2 m^{2}\left(\ln k_{\max }-\ln k_{\min }\right) \\
= & 2 k_{\min }^{2}\left(\ln k_{\max }-\ln k_{\min }\right), \\
\int_{k_{\min }}^{\infty} P(k) d k & =\frac{1}{N} \\
& \Longrightarrow \int_{k_{\max }}^{\infty} 2 m^{2} k^{-3} d k=\frac{1}{N} \\
& \Longrightarrow m^{2} k_{\max }^{-2}=k_{\min }^{2} k_{\max }^{-2}=\frac{1}{N} \\
& \Longrightarrow k_{\max }=\sqrt{N} k_{\min } .
\end{aligned}
$$

Hence, combining formula (25) and formula (26), we can get

$$
\begin{aligned}
\left\langle k^{2}\right\rangle & =2 k_{\min }^{2}\left(\ln k_{\max }-\ln k_{\min }\right) \\
& =k_{\min }^{2} \ln N .
\end{aligned}
$$


Therefore, based on the above formula (27), we can simplify formula (25):

$$
\beta_{\theta}=1+\frac{4}{\langle k\rangle \ln N}
$$

It is clear that as node average degree $\langle k\rangle$ of cluster supply chain network increases, the optimal load threshold $\beta_{\theta}$ of the whole network gradually decreases instead. Namely, the more complex the structure of cluster supply chain network is, the better robustness the network has to resist cascading failure and the stronger survivability the network has.

\section{The Process of Self-Organization Recovery}

This section introduced self-organization competition artificial neural networks into the cluster supply chain resilient recovery. Through adaptive learning, the restored nodes make preferential attachment among the old nodes and join the new round of load distribution again, which shows that the cluster supply chain resilience can achieve selforganization recovery under cascading effect.

Due to disruption, a large number of the nodes in the cluster supply chain fail and become vacant, which makes the system cannot operate normally with the combination of the remaining limited nodes. At this moment, resilient cluster supply chain as a complex adaptive system should generate new nodes on the basis of self-maintenance principle. Under the premise of effectively meeting customers' needs, the system reconstructs the logical relationship between the original remaining nodes and repaired nodes through selforganization according to the inherent constraints of supply chain and the reconstruction principle of maximizing profit, which restores the supply chain operation and ensures the system's overall profit maximization.

The intricate interaction in cluster supply chain compels them to constantly compete and collaborate, which is the basic impetus for self-organization evolution. The operation of each enterprise in cluster supply chain meets selforganization phenomenon. As there is neither centralized control mechanism nor unified command sent after the system integration, each enterprise can only consider its own survival and development and take action just based on microindividual local information. However, the spontaneous behavior of the cluster supply chain can lead to the emergence of the global structure, which makes the whole system flexible.

Neural network learning refers to the process that the network adjusts its parameters under stimulus of the external environment to make the network respond to the external environment in a new way. And its adaptability is achieved through learning, which can adjust the weights according to the environmental changes to improve system behavior.

Self-organization competition neural network integrates learning stage and working stage without providing prior standard sample in a kind of unsupervised manner. The variation of learning laws complies with the evolution equation of connection weights. It guides network learning and work based on simulating the dynamics principle where the biological neural systems process information is dependent on the action of excitement, coordination, inhibition, and competition between neurons. Through its own training, the network automatically classifies the input mode.

Fundamentals are as follows. Self-organization competition neural network has a strong self-organization adaptive learning ability. The neurons in competitive layer compete for the opportunity of response to the input mode vector, and finally only one of them becomes winner of the competition. And the winning neuron represents the classification of input mode vector. For those connection weights relevant to the winning neuron tend to be adjusted in a direction that is more conducive to competition, and the winning neuron represents the classification of input mode.

Self-organization competition neural network generally consists of input layer and competitive layer. Input layer is responsible for receiving information from outside and inputting mode and then pass them to competitive layer, which plays a role as "observation." Competitive layer is responsible for making "analysis and comparison" for the mode and finding out the law to identify the correct classification. This function is realized through the following learning mechanisms.

The learning steps of self-organization competition neural network are as follows.

(1) Initialization. Give weight vector $\omega_{i j}$ a random value within the range of $[0,1]$ according to the constraint $\sum \omega_{i j}=1$.

(2) Select the Kohonen learning rule $\Delta \omega_{i j}=\operatorname{lr}\left(p_{j}-\omega_{i j}\right)$, which corrects weights, where $p$ is the input vector, $l r$ is the learning rate.

(3) Look for the winning neuron. The weight vector that is mostly similar to the input vector is set as the competition winning neuron.

(4) Revise each connection weight that connects the winning neuron, $\omega_{i j}(t+1)=\omega_{i j}(t)+\Delta \omega_{i j}$, and other connection weights remain unchanged.

After an interval time $T$, the failure nodes are restored as new generated nodes, which select old nodes to establish connection and rejoin the load distribution according to selforganization competitive neural network learning methods. As shown in Figure 5, we assume that the failure node $v_{a}$ is restored after an interval time $T$ and the history load of all nodes as input vector. It selects the winning node from the old nodes to establish connection through using selforganization competition neural network to make classification. The nodes that already failed will naturally be eliminated in the competitive process. At this moment, select $v_{b 2}$ from adjacent node $v_{b x}(x=2,3)$ to establish connection through self-organization adaptive learning ability. Meanwhile, part of the initial load is restored:

$$
L_{i}(T)=\gamma L_{i}(0) \quad(0 \leq \gamma \leq 1) .
$$

Then this node joins the next round of cascading failure reaction process.

The ability to recover results from two parts: the characteristics that the system owns and the outside environment. 


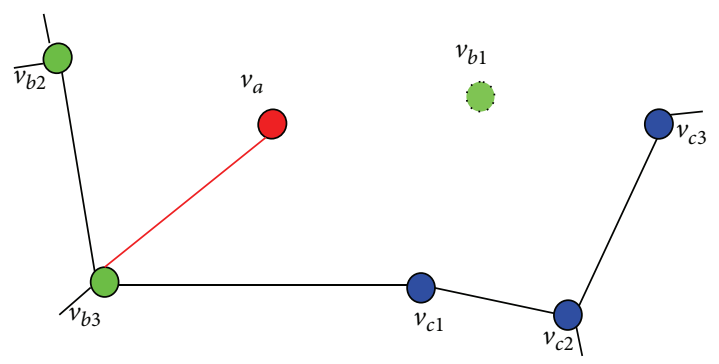

FIGURE 5: Load distribution when node is restored.

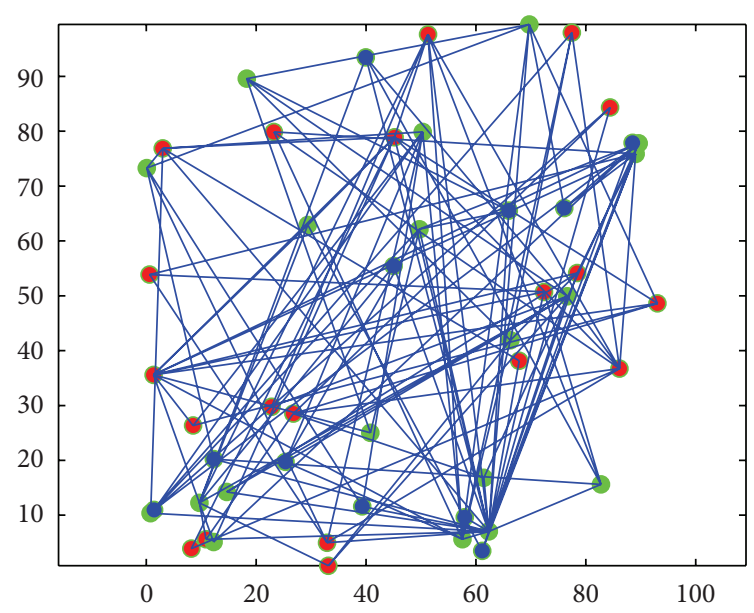

FIGURE 6: Topology structure of cluster supply chain network.

When rejoin the network, the recovery part is due to the characteristics of the system. When participating in the next round of load redistribution, the recovery part owes to the outside.

Through self-organization function of complex adaptive system, newly restored nodes reconnect the remaining nodes. Thus, cluster supply chain keeps evolving and emerges new network structure.

\section{Simulation Analysis}

Based on the cluster supply chain network structure model in Section 3.3, establish a cluster supply chain network with nodes $N=50$, and $m_{0}=5, m=3$, as shown in Figure 6 .

Take this cluster supply chain network as an example, first study the cluster supply chain network resilience without recovery mechanism, where take a node of degree 2 as the initial failure node and parameters are given as follows: $\alpha=$ $0.2, \beta=1.05$, and $\delta=1$, which means that the whole load is distributed to adjacent nodes after the node fails. As shown in Figure 6, we can see that there are multiple occurrences of horizontality in the curve, which reflects the absorption capacity that the cluster supply chain network has. Although cascading reaction carries on continuously, owing to the complementarities of cluster supply chain network that adjacent nodes can share the capacity of the failure node, the overall performance does not decline within a period. But this method, which only considers the cascading failure without

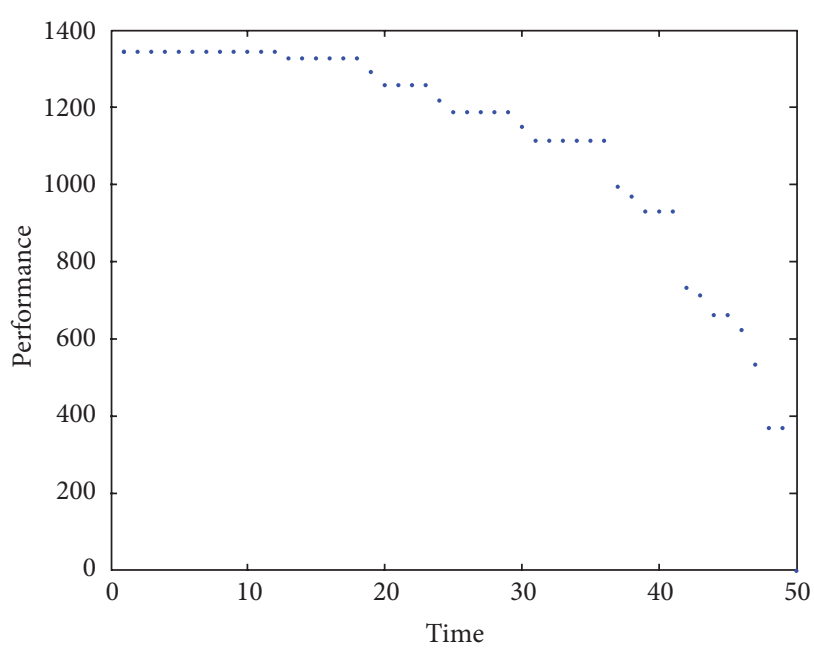

FIGURE 7: Resilience of cluster supply chain without recovery mechanism.

considering node recovery, will make the entire cluster supply chain network eventually collapse.

Adopt the approach with self-organization recovery mechanism to study the same cluster supply chain network resilience, where $\gamma=0.6$. When a node is restored, as shown in Figure 7, the situation depicted in Figure 1 will occur repeatedly. Meanwhile, the network performance will undulate down over time and ultimately reach a new steady state. The forward process with undulating style reflects the adaptability and recovery ability that the cluster supply chain network possesses. The node in the network can actively respond to its failure and transfer its load to the neighbors. That behavior belongs to self-organization ability, which makes the network performance decline slowly. The failure nodes are restored after a period of time and take the use of self-organization competition neural network to make classification and select the winning node in the old nodes to establish a connection and rejoin the network. At this moment, the ability of the failure nodes is partly restored but can once again participate in sharing the load distributed by the failure adjacent nodes. The recovery ability that combines both the internal and external part makes the network performance rebound in a period of time.

Comparing Figure 7 to Figure 8, we can get Figure 9. It is clear that the cluster supply chain with self-organization recovery mechanism is more coincident with actual situation and can ultimately reach a steady state through this dynamic process. In order to achieve the original state or better state, it needs more investment than simple repair cost. The reasonable selection of parameters $\delta, \gamma$ reflects network adaptability and is also the key to the degree of network resilience.

Emergence is a kind of transition from low level to high level based on the evolution of the microbody, referring to the mutation of the macrosystem in performance and structure. The phenomenon of emergence is centered on the interaction and is more complex than simple summation of individual behavior. Cluster supply chain failure and recovery have the characteristics of emergence. 


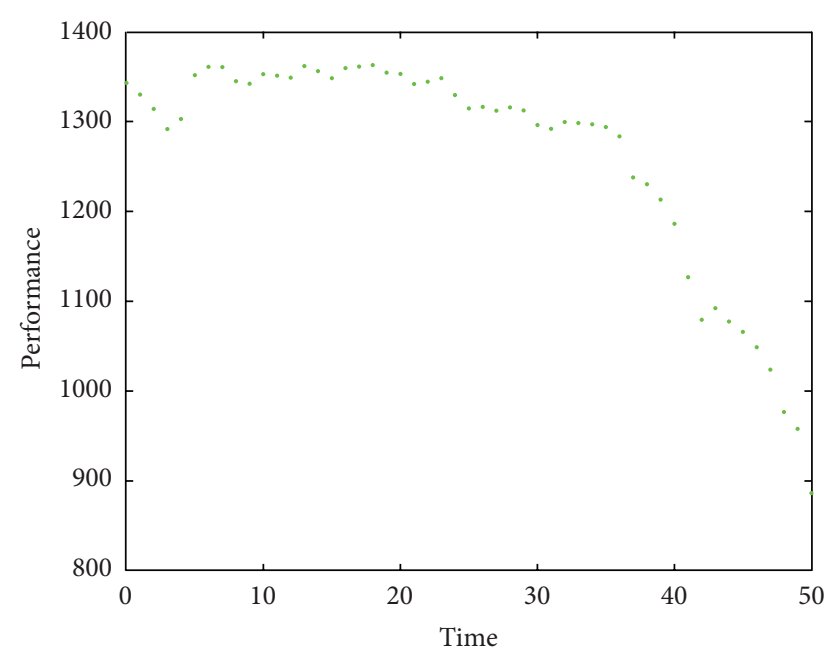

FIGURE 8: Resilience of cluster supply chain with self-organization recovery mechanism.

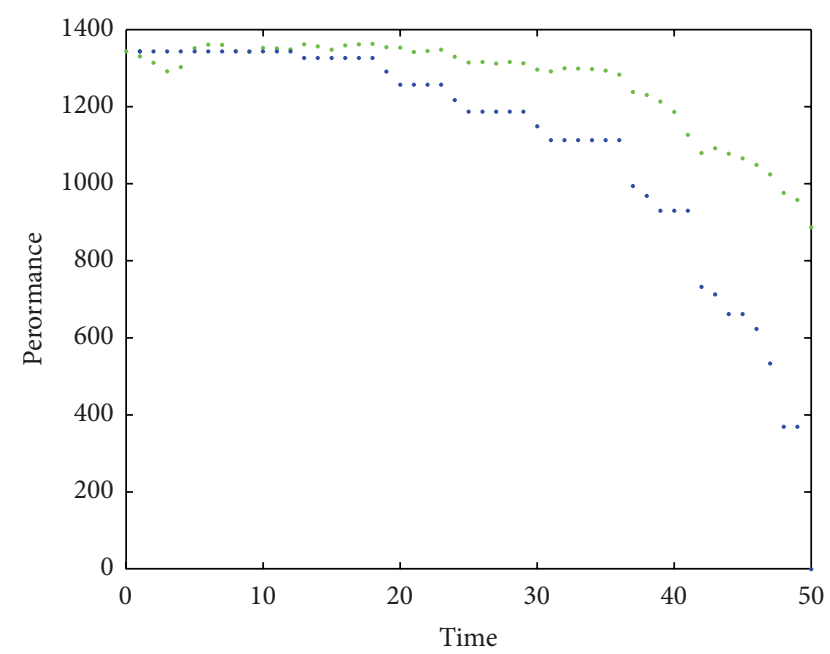

FIGURE 9: Comparison of whether recovery mechanism exits or not.

By contrast, we can observe the evolution phenomenon of local effect and the emergence of structure and function. Each node enterprise can make the entire cluster supply chain achieve optimization through local self-organization learning, which is reason why the cluster supply chain has resilience.

\section{Conclusions}

With the rapid economic development, the changes of both cluster supply chain external environment and its internal operations become intense, which leads to the frequent occurrence of interrupt events. The cluster supply network with better resilience can withstand various interrupts, and it has a relatively shorter interrupt recovery time and stronger competitiveness. The major contributions of the research are as follows.
(1) Based on the literature review, the concept of cluster supply chain resilience is put forward, which includes such basic points as absorption capacity, adaptability, and recovery ability. In addition, the expression of resilience is also developed.

(2) The generation of cluster supply chain network structure is well illustrated, which takes its scale-free property into account.

(3) The cascading failure model is developed to illustrate the dynamic evolution process of failure under interrupt environment. Besides, we make full analysis of how this network structure affects anti-disruption, which shows that the greater the node average degree is, the stronger anti-disruption the network has.

(4) The self-organization property of cluster supply chain resilience is fully elaborated. With respect to the selforganization recovery ability, it is shown through simulation. Meanwhile, it is found out that cascading failure is the root of cluster supply chain vulnerability while self-organization is the key to cluster supply chain resilient recovery. In addition, the emergence property that failure and recovery owns is clearly identified, which can serve as theoretical guidance and reference for achieving overall resilience optimization through local control.

The future study will focus on how to improve the adaptability that each node possesses. After that, how to optimize the cluster supply chain resilience so as to provide favorable basis for dealing with supply chain network interrupt also needs further study.

\section{Acknowledgment}

This project is supported by the National Natural Science Foundation of China (no. 71171089).

\section{References}

[1] G. Sun, "Research on the fresh agricultural product supply chain coordination with supply disruptions," Discrete Dynamics in Nature and Society, vol. 2013, Article ID 416790, 9 pages, 2013.

[2] C. S. Holling, "Resilience and stability of ecological systems," Annual Review of Ecology and Systematics, vol. 4, pp. 1-23, 1973.

[3] S. Anderson, "Business + IT=Resilience," Contingency. Planning \& Management, vol. 78, no. 5, pp. 236-245, 2003.

[4] M. Christoper and H. Pack, "Building the resilient supply chain," The International Journal of Logistics Management, vol. 15, no. 2, pp. 1-13, 2004.

[5] J. Fiksel, "Sustainability and resilience: toward a systems approach," Sustainability, vol. 2, no. 2, pp. 14-21, 2006.

[6] J. Rice and F. Caniato, "Building a secure and resilient supply network," Supply Chain Management Review, vol. 7, no. 5, pp. 22-31, 2003.

[7] Y. Sheffi, "Building a resilient supply chain," Harvard Business Review, vol. 1, no. 8, pp. 1-4, 2005.

[8] Y. Sheffi, "Building a resilient organization," The Bridge Engineering for the Threat of Natural Disasters, vol. 37, no. 1, pp. 1-17, 2007. 
[9] K. Zhao, A. Kumar, T. P. Harrison, and J. Yen, "Analyzing the resilience of complex supply network topologies against random and targeted disruptions," IEEE Systems Journal, vol. 5, no. 1, pp. 28-39, 2011.

[10] Y. Huang, Z. Zhang, and X. Zhang, "Assessment and management vulnerability of supply networks structure based on social networks analysis," in Proceedings of the 2nd IEEE International Conference on Advanced Management Science (ICAMS '10), pp. 128-132, July 2010.

[11] Y. Yan, X. Liu, and X.-T. Zhuang, "Resilient supply chain emergency management strategy based on node fails," Control and Decision, vol. 25, no. 1, pp. 25-30, 2010.

[12] E. D. Vugrin, D. E. Warren, and M. A. Ehlen, "A resilience assessment framework for infrastructure and economic systems: quantitative and qualitative resilience analysis of petrochemical supply chains to a hurricane," Process Safety Progress, vol. 30, no. 3, pp. 280-290, 2011.

[13] W. Wang, W. Fu, G. Wang, and L. Hao, "Complex dynamics of supply chain system with learning ability," Journal of Mechanical Engineering, vol. 47, no. 8, pp. 175-182, 2011.

[14] Y. P. Li, W. H. Zhang, S. Wang et al., "Supply chain super-network model and area resources planning of industrial cluster," Journal of Shenyang University (Natural Science), vol. 24, no. 6, pp. 85-88, 2012.

[15] Z. P. Zuo and J. Z. Li, "Research on the combination mechanism of cluster supply chain and Eco-industrial Park," Forum on Science and Technology in China, vol. 5, pp. 137-156, 2012.

[16] C.-H. Lin, C.-M. Tung, and C.-T. Huang, "Elucidating the industrial cluster effect from a system dynamics perspective," Technovation, vol. 26, no. 4, pp. 473-482, 2006.

[17] B. He and G. Song, "Cluster supply chain network structure and knowledge transfer," in Proceedings of the International Conference on Information Management, Innovation Management and Industrial Engineering (ICIII '09), vol. 3, pp. 615-618, December 2009.

[18] Q. Wu, "Cluster supply chain risk and its governance," Applied Mechanics and Materials, vol. 63-64, pp. 260-263, 2011.

[19] X. Li and G. Chen, "A local-world evolving network model," Physica A, vol. 328, no. 1-2, pp. 274-286, 2003.

[20] J.-F. Zheng, Z.-Y. Gao, and X.-M. Zhao, "Modeling cascading failures in congested complex networks," Physica A, vol. 385, no. 2, pp. 700-706, 2007.

[21] J.-W. Wang and L.-L. Rong, "Robustness of the western United States power grid under edge attack strategies due to cascading failures," Safety Science, vol. 49, no. 6, pp. 807-812, 2011.

[22] J. Winkler, L. Dueñas-Osorio, R. Stein, and D. Subramanian, "Interface network models for complex urban infrastructure systems," Journal of Infrastructure Systems, vol. 17, no. 4, pp. 138150, 2012.

[23] S. Wang, L. Hong, and X. Chen, "Vulnerability analysis of interdependent infrastructure systems: a methodological framework," Physica A, vol. 391, no. 11, pp. 3323-3335, 2012. 


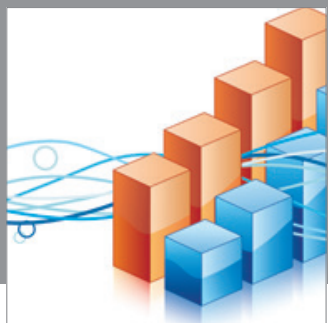

Advances in

Operations Research

mansans

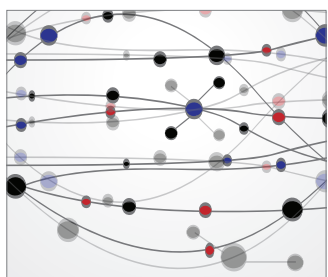

The Scientific World Journal
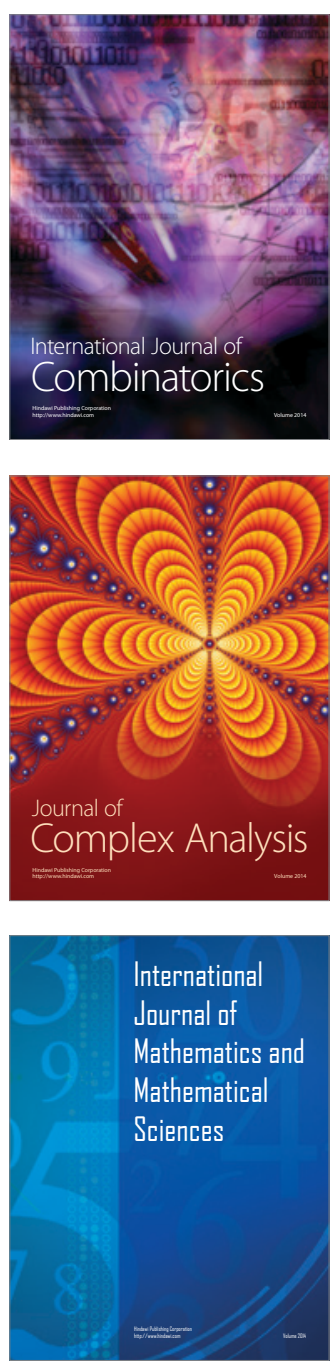
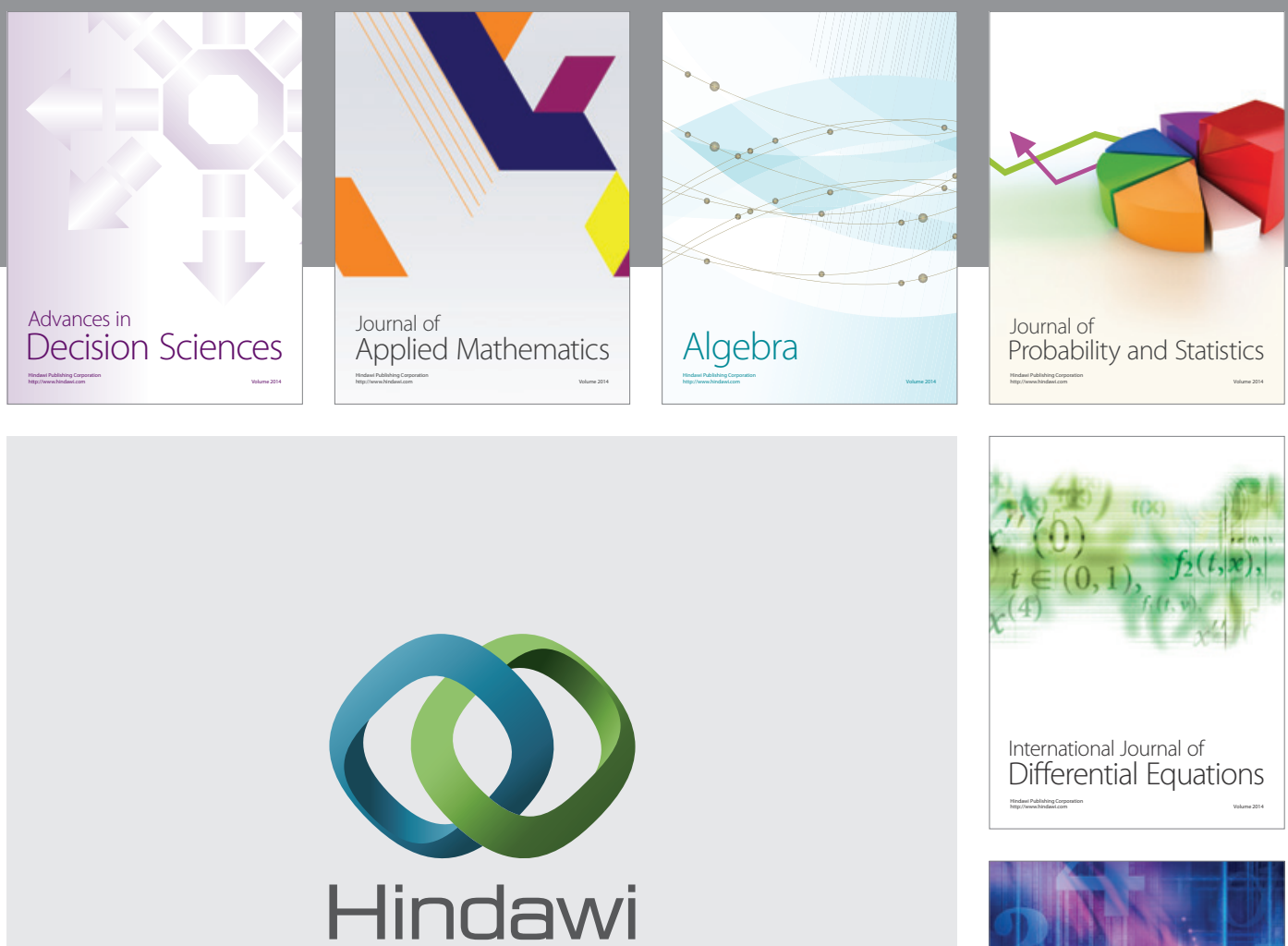

Submit your manuscripts at http://www.hindawi.com
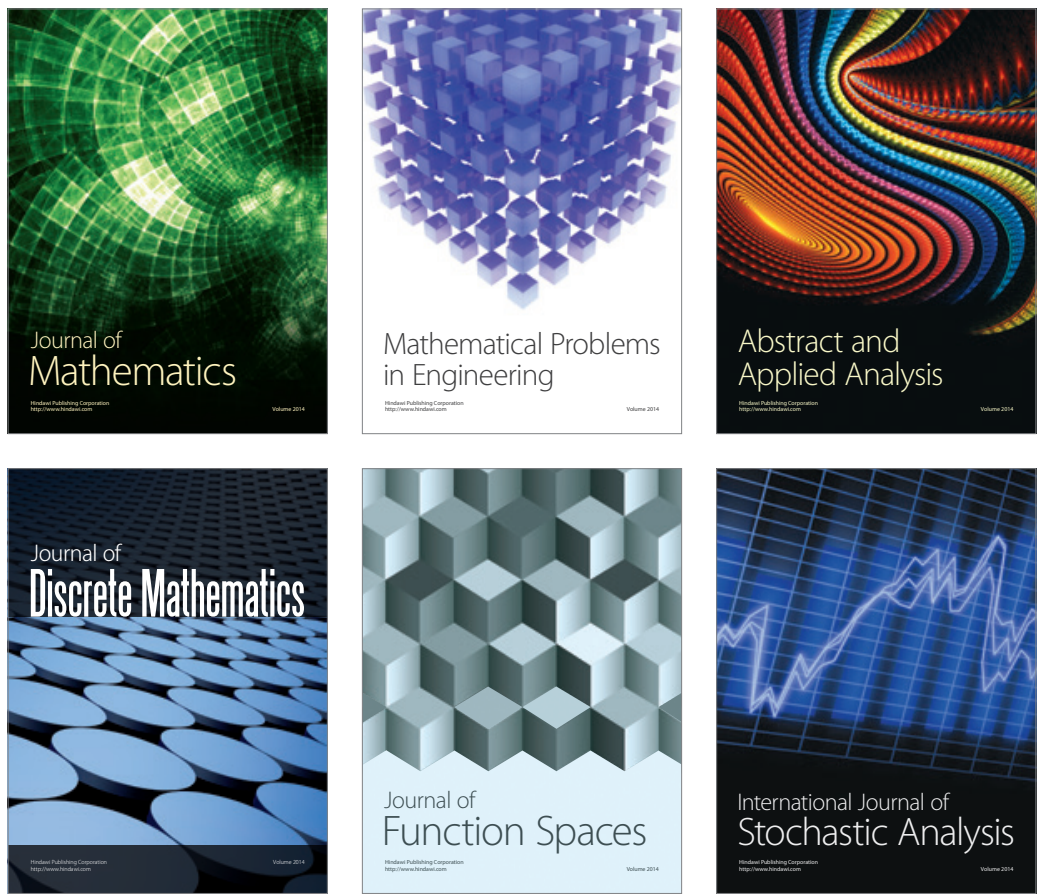

Journal of

Function Spaces

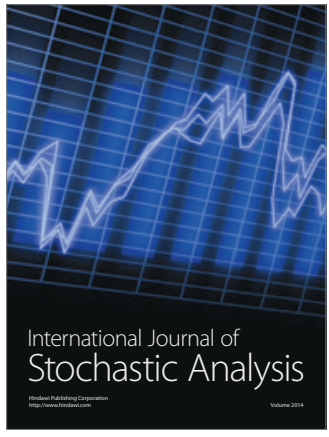

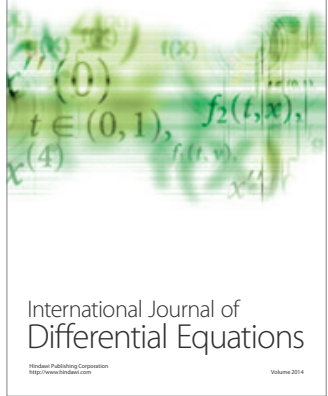
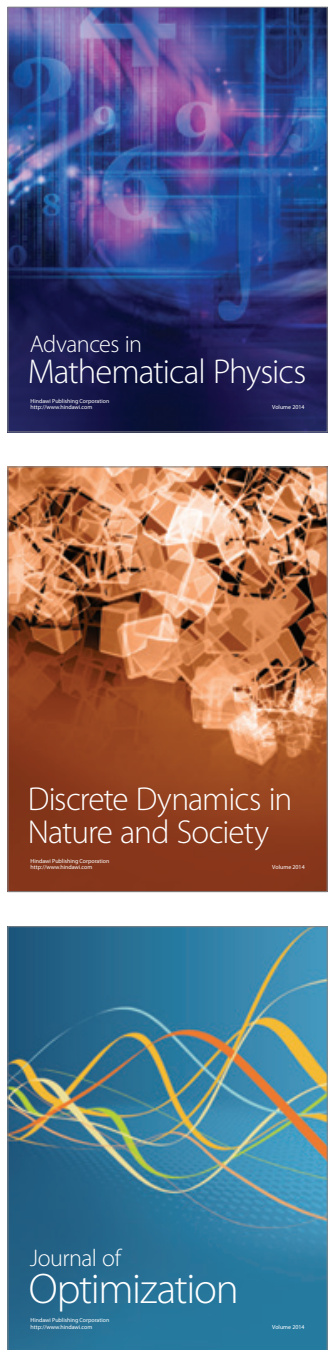